\title{
PAEDIATRICS
}

\section{Superglues or sutures-fistula repairs that stick}

The results of a randomized clinical trial suggest that application of a cyanoacrylate adhesive is a suitable alternative to surgery, which is the current gold standard of treatment for urethrocutaneous fistulae. The two approaches have equivalent success rates, but the use of cyanoacrylate is simple and inexpensive.

Hypospadias, a common birth defect affecting placement of the urethral meatus, is corrected using urethroplasty. Although a variety of surgical techniques is available, complications from correction of hypospadias are common. Urethrocutaneous fistulae have an estimated incidence of 35\% following hypospadias repair in males. Surgical intervention is required to close the fistulae, but closure often requires multiple operations, with a 6-12 month interval between each procedure.

Cyanoacrylate glues can be used as alternatives to sutures for wound closure. Monomeric cyanoacrylates rapidly polymerize on exposure to air to form a solid acrylic resin with high tensile strength. The resin adheres to epithelial surfaces for 7-10 days, creating a waterproof layer with bacteriostatic and haemostatic properties.

\section{4 ...surgery might not always be the gold standard of treatment... 77}

On the basis of previous studies suggesting potential benefits of the use of cyanoacrylate adhesives in the management of urethrocutaneous fistulae, AmbrizGonzález and colleagues have conducted a randomized trial to compare this approach with traditional surgical repair. 42 boys $<7$ years old, with one or more fistulae $<5 \mathrm{~mm}$ in diameter following surgical treatment for hypospadias, were enrolled and randomly assigned to closure of the fistulae by surgery or by application of 2-octyl cyanoacrylate.

Preparation for adhesive treatment involved application of anti-inflammatory corticosteroid cream for 3 days, and then sedation and Foley catheterization in an outpatient setting. Adhesive was applied in several thin layers following gentle scarification and approximation of the edges of the fistula. Closure was evaluated after 5 days, and if a fistula persisted, adhesive was applied up to twice more. If a fistula was not closed after three applications, the procedure was deemed to have failed.

17 of 28 fistulae occurring in 21 patients were successfully closed, using an average of 2.5 applications of $1 \mathrm{ml}$ of 2-octyl cyanoacrylate. 25 fistulae were treated in 21 patients in the surgical arm, and 17 closures were achieved with an average of 3.2 surgeries per patient. The use of cyanoacrylate reportedly resulted in a number needed to treat of 12 to prevent a surgical intervention. Furthermore, the total cost of treatment with cyanoacrylate was calculated as being equivalent to US $\$ 14,809$, compared with $\$ 158,538$ for surgery and associated hospital stays.

Growing evidence for the benefits of cyanoacrylate closure of urethrocutaneous fistulae suggests that surgery might not always be the gold standard of treatment for this common paediatric condition. For young children (and their parents), following hypospadias repair, avoiding further hospital stays has clear advantages.

\section{Robert Phillips}

Original article Ambriz-González, G. et al. 2-octyl cyanoacrylate versus reintervention for closure of urethrocutaneous fistulae after urethroplasty for hypospadias: a randomized controlled trial. BMC Urol. doi:10.1186/1471-2490-14-93

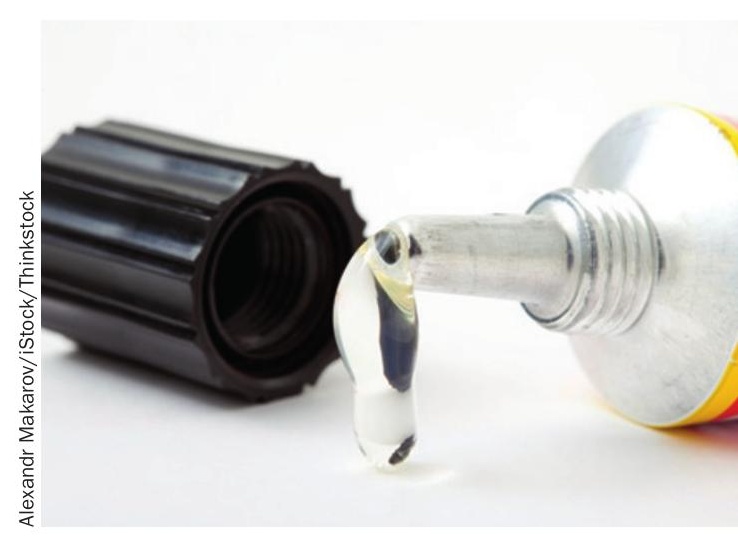

